Supporting Information for

\title{
Robust Nanoparticle-DNA Conjugates Based on Mussel-Inspired Polydopamine Coating for Cell Imaging and Tailored Self-Assembly
}

Chenxu Wang, ${ }^{\dagger}$ Jiajing Zhou, ${ }^{\dagger}$ Peng Wang, ${ }^{\dagger, \star}$ Wenshan He, ${ }^{\S,}{ }^{*}$ Hongwei Duan ${ }^{\dagger, *}$

${ }^{\dagger}$ School of Chemical and Biomedical Engineering, Nanyang Technological University, 70 Nanyang Drive, Singapore 637457.

Nanyang Environment and Water Research Institute (NEWRI), Nanyang Technological University, 1 Cleantech Loop, Singapore 637141.

${ }^{\S}$ Union Hospital, Tongji Medical College, Huazhong University of Science \& Technology, Wuhan 430022, P. R. China

Email: hduan@ntu.edu.sg,hewenshan@hust.edu.cn 


\section{Table of Contents:}

Scheme S1. The conjugation of PDA-coated substrates with molecules containing nucleophilic amine or thiol groups via Michael addition and/or Schiff base reaction...............3

Figure S1. TEM images of 50 nm AuNPs and $\mathrm{Au}_{50} @$ PDA-5 ................................................. 3

Figure S2. UV-vis absorption spectra of $\mathrm{Au}_{50} @ \mathrm{PDA}$ with $5 \mathrm{~nm}$ shell................................... 4

Figure S3. Dark field images of 50 nm AuNPs and $\mathrm{Au}_{50} @$ PDA-5 ...................................... 4

Figure S4. Scattering spectrum of $\mathrm{Au}_{50} @$ PDA with $5 \mathrm{~nm}$ shell ........................................... 5

Figure S5. TEM images of Au50@PDA-8 separated from 160 mM NaCl dispersion ............5

Figure S6. Electrophoresis migration of Au50@PDA-8, Au50@PDA-8 modified with thiolended A1, and Au50@PDA-8 with A2 without a thiol end group ........................................ 6

Figure S7. The pH stability of Au50@PDA-8@DNA1 at pH 2-12..................................... 6

Figure S8. Cytotoxicity tests of Au50@PDA-8@DNA and Au50@PDA-8............................7

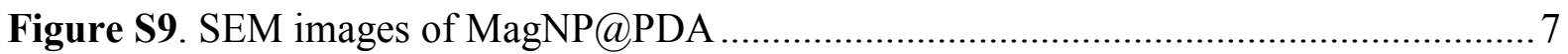



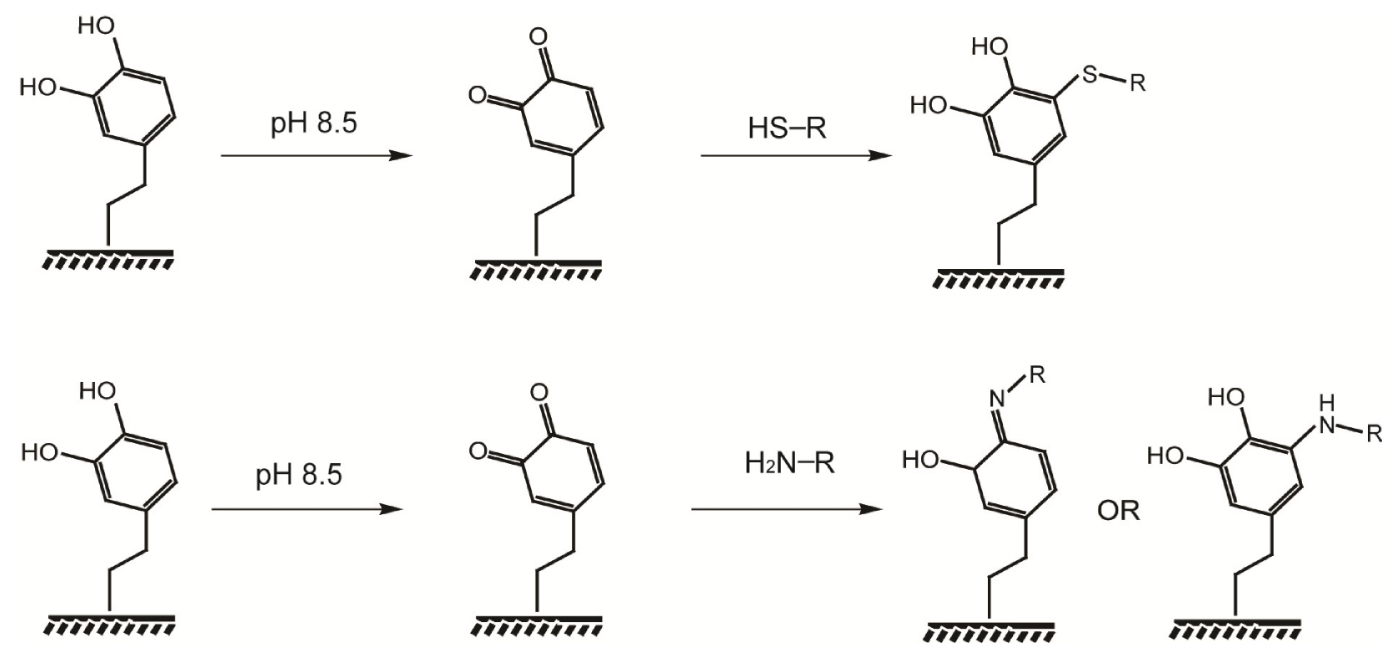

Scheme S1. The conjugation of PDA-coated substrates with molecules containing nucleophilic amine or thiol groups via Michael addition and/or Schiff base reaction.
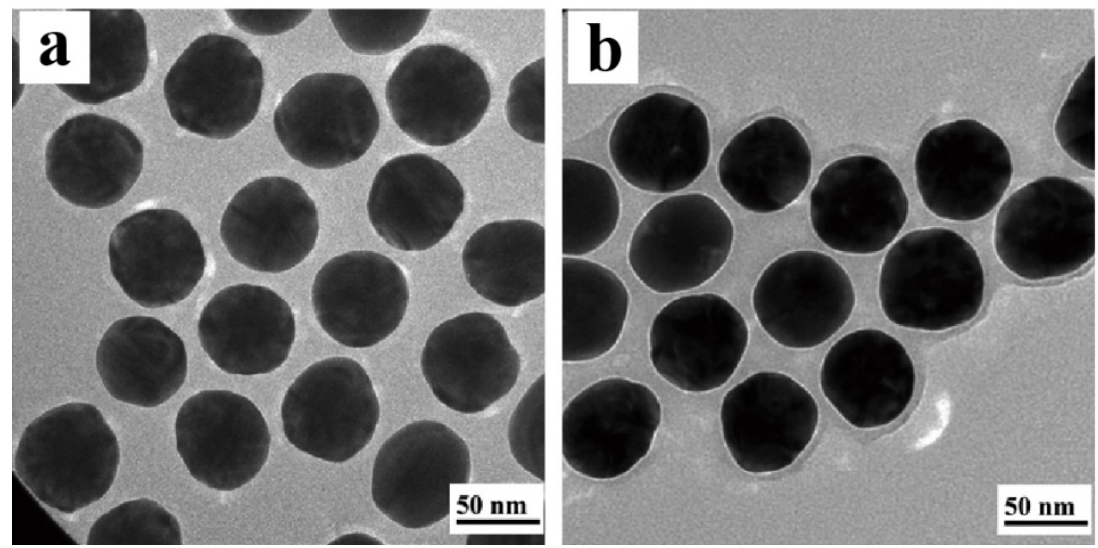

Figure S1. TEM images of (a) $50 \mathrm{~nm}$ AuNPs and (b) Au $\mathrm{u}_{50} @$ PDA-5. 


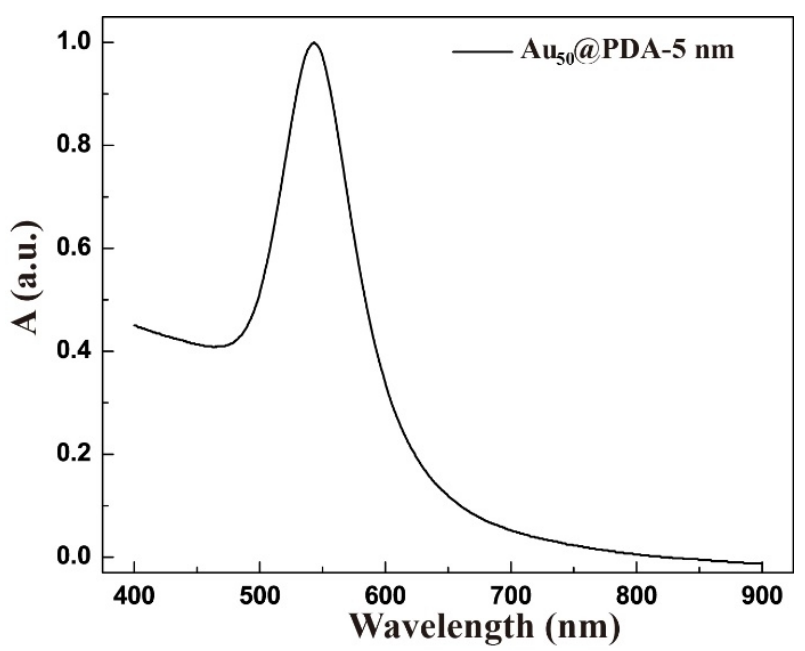

Figure S2. UV-vis absorption spectra of $A_{4}{ }_{50} @ P D A$ with 5 nm shell.
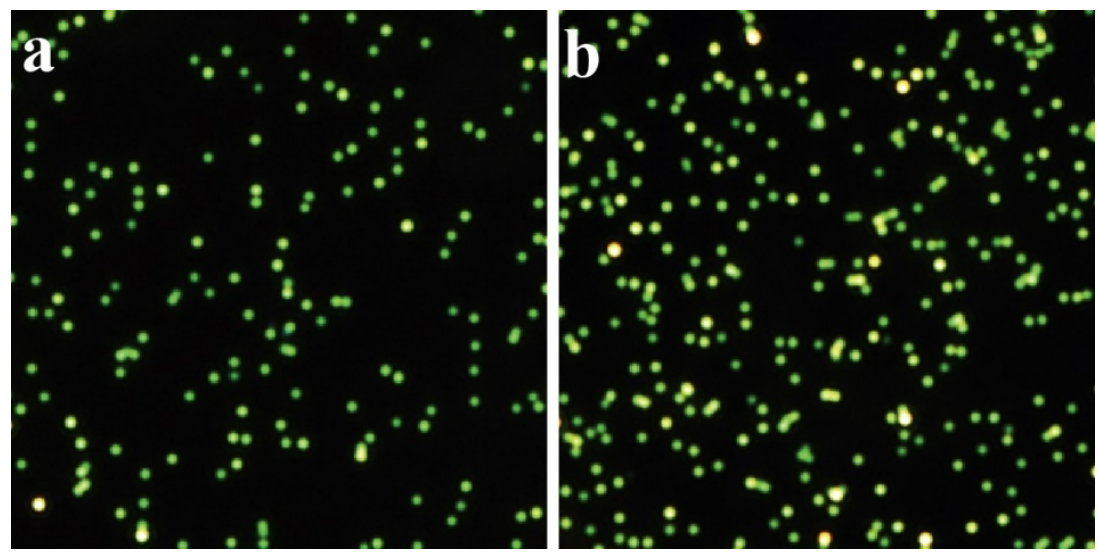

Figure S3. Dark field image of (a) 50 nm AuNPs and (b) Au ${ }_{50} @$ PDA-5. 


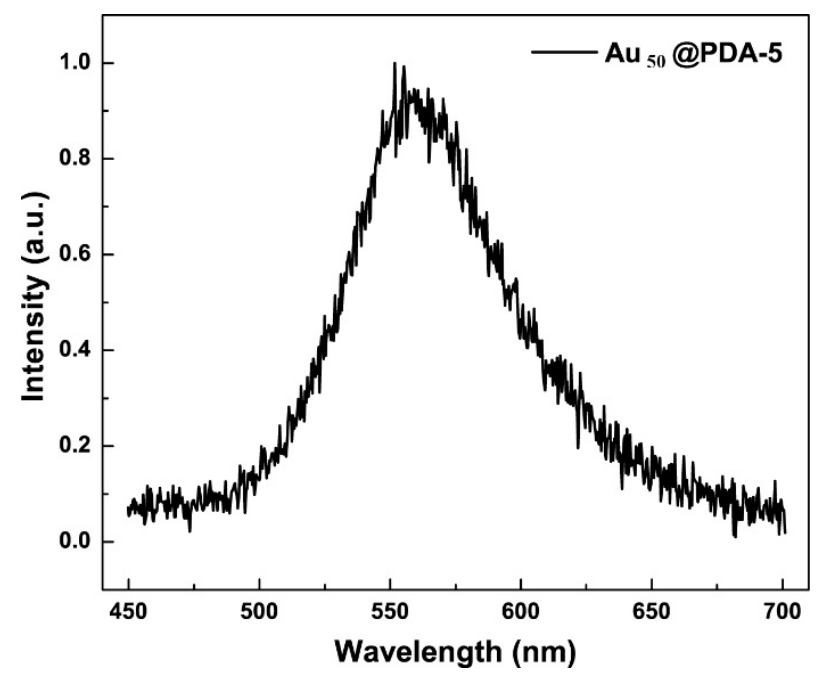

Figure S4. Scattering spectra of $\mathrm{Au}_{50} @$ PDA with 5 nm shell.

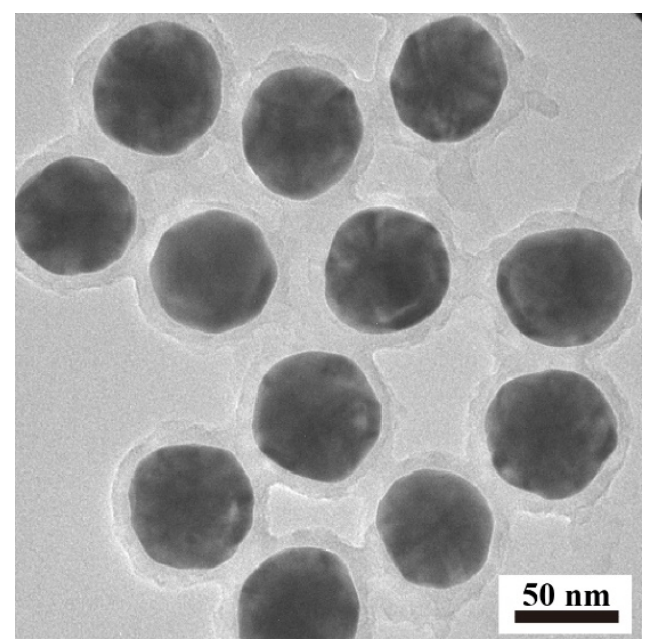

Figure S5. TEM images of Au50@PDA-8 separated from 160 mM NaCl dispersion. 


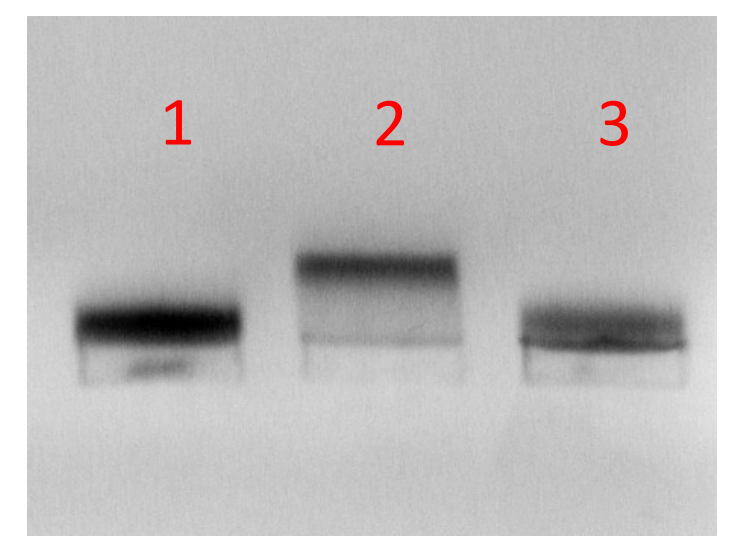

Figure S6. Electrophoresis migration of Au50@PDA-8 (band 1), Au50@PDA-8 modified with thiol-ended A1 (band 2), and Au50@PDA-8 with A2 without a thiol end group (band 3). DNA sequences of A1 and A2 are listed below.

$\begin{array}{cc}\text { ssDNA } & \begin{array}{c}\text { Sequence (5'-3') } \\ \text { A1 }\end{array} \\ \text { A2 } & \text { T-S--TTTTTTTTTTT-ACC TGG GGG AGT ATT GCG GAG GAA GGT-Cy5 } \\ \text { TTTTTT-ACC TGG GGG AGT ATT GCG GAG GAA GGT-Cy5 }\end{array}$

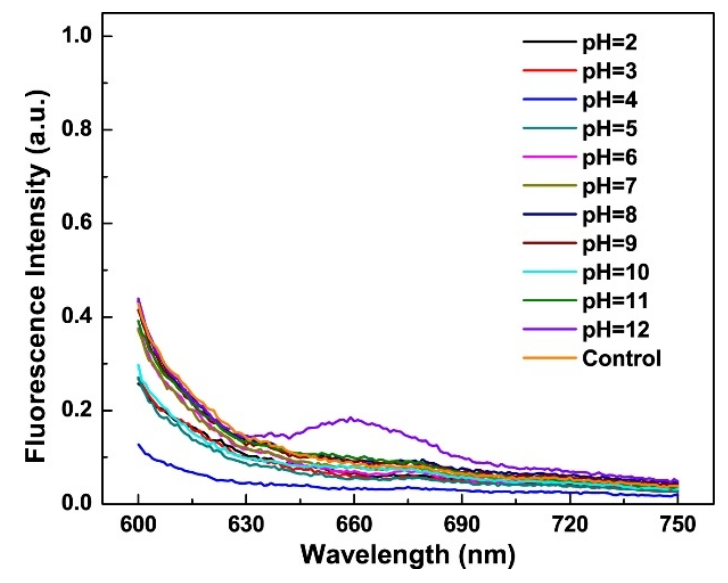

Figure S7. The pH stability of Au50@PDA-8@DNA1 at pH 2-12. 

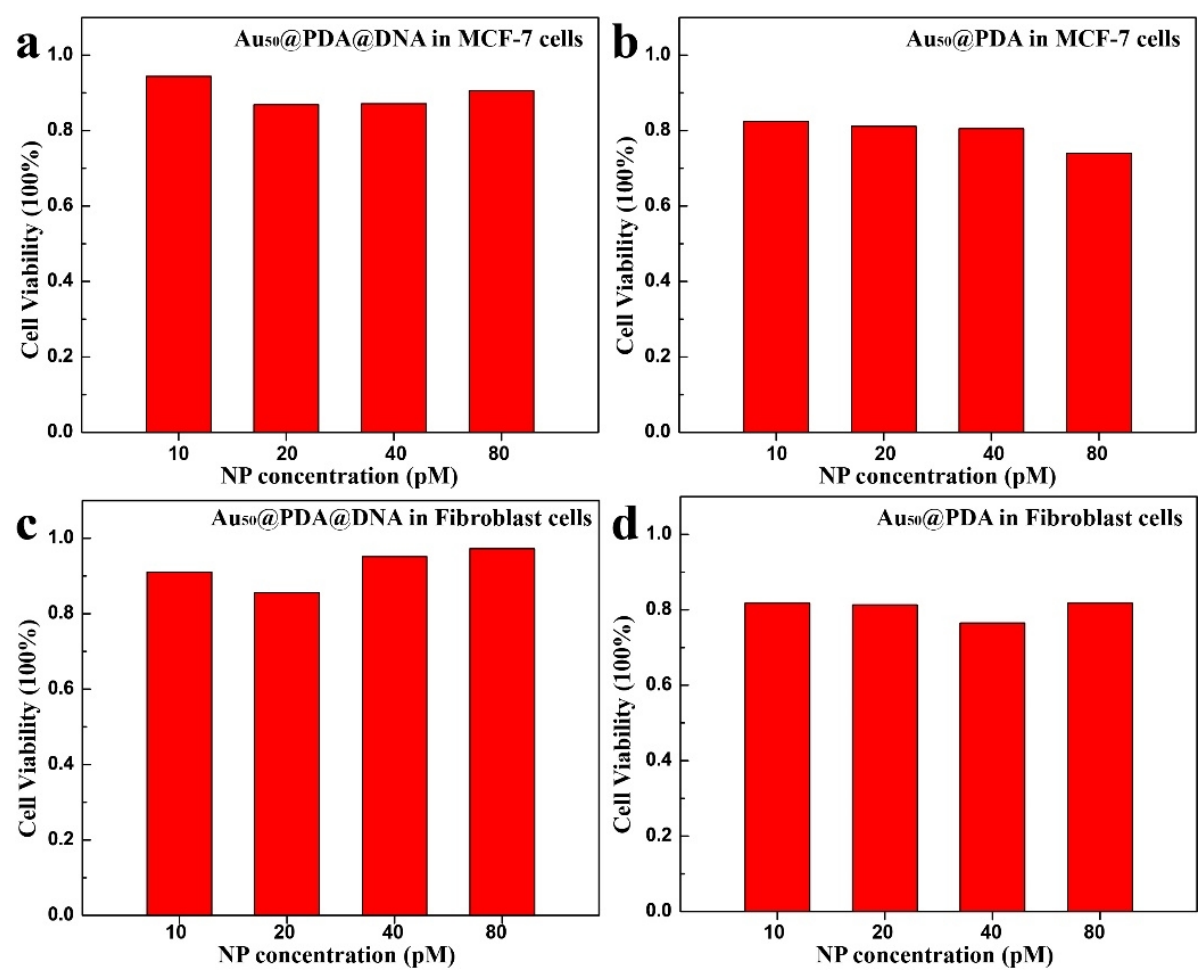

Figure S8. Cytotoxicity tests of Au50@PDA-8@DNA and Au50@PDA-8. MCF-7 cells treated with Au50@PDA-8@DNA (a) Au50@PDA-8 (b). NIH 3T3 cells treated with Au50@PDA-8@DNA (c) and Au50@PDA-8 (d).

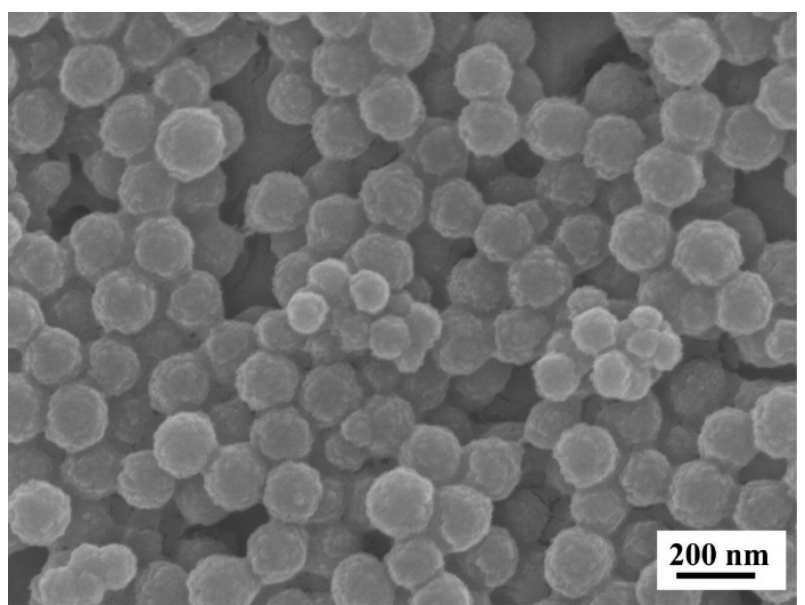

Figure S9. SEM images of MagNP@PDA. 